\title{
A Research on Nonlinear Frequencies of Multibody Contact Systems
}

\author{
Yao Tingqiang \\ School of Mechanical and Eelectric \\ Engineering \\ Kunming University of Science and \\ Technology \\ Kunming, China \\ yaotingqiang@163.com
}

\author{
Tang Xiuying \\ Faculty of Engineering and \\ Technology, Yunnan Agricultural \\ University, Kunming, China \\ tangxiuying@126.com
}

\author{
Chi Yilin \\ School of Mechanical and Eelectric \\ Engineering \\ Kunming University of Science and \\ Technology \\ Kunming, China \\ yilinchi@kmust.edu.cn
}

\begin{abstract}
The paper has discussed the effect of dynamic contact behaviour on dynamics characteristics and contact nonlinear frequencies of multibody systems with geometric, physical and load parameters and motion conditions. In the work presented, a linearization method for contact nonlinear frequencies of constrained multibody systems is investigated. The nonlinear equations of motion of multibody systems are expressed indirectly in terms of the variations of independent coordinates, velocities and accelerations. The Lagrange multipliers are eliminated to get linearized equations of motion. The simulation analysis of kinematics, dynamics and contact nonlinear frequencies of numerical examples have been solved simultaneously. It shows that the frequencies of multibody systems with dynamic contact behaviours are nonlinear, timevarying and periodic with the effect of load-deformation factor, contact stiffness, load, motion conditions and initial conditions. This linearization method is available to research the contact nonlinear frequencies of rolling bearings.
\end{abstract}

Keywords- Contact nonlinear frequency;contact dynamics; dynamics analysis;dynamics of multibody systems

\section{INTRODUCTION}

There are three nonlinearity such as material nonlinearity, geometric nonlinearity and contact nonlinearity in multibody systems. The contact nonlinearity is also called state nonlinearity. The constrainted multibody systems usually have contact nonlinear dynamics characteristics and vibration response, which are affected by dynamic contact behavior between the parts with different geometric parameters, physical parameters, motion conditions and load parameters[1-4]. As known the rolling bearings are typical multibody systems, which have multibody contact dynamics behaviour and nonlinear vibration response. The exibility of the rolling contacts has always been represented by nonlinear springs and their stiffness are obtained by Hertz theory in the quasi-dynamics models of rolling bearings[5-9]. This paper discusses a linearization method for contact nonlinear frequencies of constrained multibody systems considering dynamic contact behaviours. The structure of the paper is as follow: First introduce the literature, the mathematical model is established, then a linearization method for the constrained multibody systems has been investigated, finally, dynamics analysis and contact nonlinear frequencies of numerical examples are discussed by the method.
Recently, nonlinear frequency response analysis has become one of the most interested research based on nonlinear dynamics theory. Sohoni[10] presented an approach for automatically generating a linearized dynamical model, which is derived from the nonlinear equations of motion. The Lagrange multiplier term was kept constant in the linearized equations of motion. The velocity and acceleration level constraints have not been considered in the resulting linearized equations of motion. Neuman symbolically generated the dynamic robot model by Lagrange-Euler formulation and linearized the dynamic model about a nominal trajectory[11]. Balafoutis presented a computational method for recursive evaluation of linearized dynamic robot model about a nominal trajectory[12]. The formulation was applied to the robot systems, which are unconstrained systems. The formulation was applied to the robot systems, which are unconstrained systems. This formulation was generalized by Gontier[13] for general unconstrained mechanical systems. The equations of motion of a multibody system are linearized and reduced to independent coordinates, using an orthogonal complement method by Minaker[14]. Lynch presented a method for obtaining linearized state space representations of open or closed loop multibody dynamic systems[15]. The pre-stress modal analysis method is applied widely to calculate the frequency response of few parts considering contact nonlinearity by FEM in ANSYS or other FEM commercial softwares[16]. This method can solve some nonlinear frequency with static and weak nonlinear contact, small displacements and deformation between few relative simple geometric parts. However, there are constraints and corresponding Lagrange multipliers, large displacements or deformation, strong nonlinear contact, dynamic contact-impact behavior and discontinuous contact with clearance in multibody systems. The pre-stress modal analysis method can not solve frequency response of multibody systems accurately, sometimes, it is impossible and incorrect.

\section{EQUATIONS OF MOTION}

A linearization method for the constrained multibody systems has been investigated in the work presented. Based on a relative simple contact dynamics model with multibody dynamic contact, large displacements and constraints, as show in Fig1, the dynamics characteristics and contact 
nonlinear vibration response of multibody systems have been discussed.The equations of motion for the model in Fig. 1 can be written as

$$
\left\{\begin{array}{c}
\boldsymbol{M} \ddot{\boldsymbol{q}}=\boldsymbol{F}-\boldsymbol{\Phi}_{q}^{\mathrm{T}} \lambda \\
\boldsymbol{\Phi}=0
\end{array}\right.
$$

where $\boldsymbol{M}$ is total mass matrix, $\boldsymbol{q}$ is generalized coordinate vector, $\boldsymbol{F}$ is generalized loads(nonlinear contact forces $F_{c}=K_{c} \delta^{n}+\operatorname{step}\left(\delta, 0,0, \delta_{\max }, c_{0}\right) \cdot \dot{\delta}, K_{c}$ is contact stiffness, $\delta$ is contact elastic deformation, $n$ is loaddeformation factor, $c_{0}$ is constant damping, step $(. .$.$) is step$ function), $\boldsymbol{\Phi}$ is constraint equations, $\lambda$ is Lagrange multipliers. The equations of motion for multibody systems are highly nonlinear with respect to the positions, velocities, and accelerations[2,17]. It's much more difficult to calculate the frequency response directly in Eq.(1) due to the constraints, Lagrange multipliers and contact force.

The independent and dependent coordinates, velocities, and accelerations are tightly and nonlinearly coupled by the position, velocity, and acceleration level constraints. The equations of motion are implicit function of the coordinates, velocities, and accelerations. As a result, it is to only express the equations of motion in terms of the independent coordinates, velocities, and accelerations.

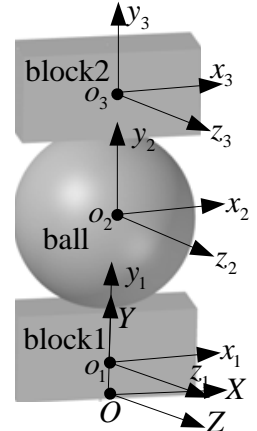

(a)3D simulation mode Fig1.The contact dynamics model

\section{A LINEARIZATION METHOD FOR CONSTRAINTED MULTIBODY SYTEMS}

The coordinates $\boldsymbol{q}$ can be partitioned into dependent coordinates $\boldsymbol{q}_{\mathrm{D}}$ and independent coordinates $\boldsymbol{q}_{\mathrm{I}}$ such that the sub-Jacobian $\boldsymbol{\Phi}_{\boldsymbol{q}_{\mathrm{D}}}$ is well conditioned. Variational form of the constraint equations can be written as

$$
\delta \boldsymbol{\Phi}=\boldsymbol{\Phi}_{q_{\mathrm{D}}} \delta \boldsymbol{q}_{\mathrm{D}}+\boldsymbol{\Phi}_{q_{\mathrm{I}}} \delta \boldsymbol{q}_{\mathrm{I}}=0
$$

The relationship between $\boldsymbol{q}_{\mathrm{D}}$ and $\boldsymbol{q}_{\mathrm{I}}$ can be written as

$$
\delta \boldsymbol{q}_{\mathrm{D}}=-\boldsymbol{\Phi}_{q_{\mathrm{D}}}^{-1} \boldsymbol{\Phi}_{q_{\mathrm{I}}} \delta \boldsymbol{q}_{\mathrm{I}}
$$

Using the relationship in Eq.(3), $\delta \boldsymbol{q}$ is represented as

$$
\begin{gathered}
\text { where } \boldsymbol{N}=\left[\begin{array}{c}
-\boldsymbol{\Phi}_{\boldsymbol{q}_{\mathrm{D}}}^{-1} \boldsymbol{\Phi}_{\boldsymbol{q}_{\mathrm{I}}} \\
\boldsymbol{I}
\end{array}\right]=\left[\begin{array}{c}
\delta \boldsymbol{q}_{\mathrm{D}} \\
\delta \boldsymbol{q}_{\mathrm{I}}
\end{array}\right]=\left[\begin{array}{c}
-\boldsymbol{\Phi}_{\boldsymbol{q}_{\mathrm{D}}}^{-1} \boldsymbol{\Phi}_{\boldsymbol{q}_{\mathrm{I}}} \\
\boldsymbol{I}
\end{array}\right] \delta \boldsymbol{q}_{\mathrm{I}}=\boldsymbol{N} \delta \boldsymbol{q}_{\mathrm{I}}(4) \\
\boldsymbol{N}^{\mathrm{T}} \boldsymbol{\Phi}_{\boldsymbol{q}}^{\mathrm{T}}=\left[\begin{array}{ll}
-\boldsymbol{\Phi}_{\boldsymbol{q}_{\mathrm{D}}}^{-1} \boldsymbol{\Phi}_{\boldsymbol{q}_{\mathrm{I}}} & \boldsymbol{I}
\end{array}\right]\left[\begin{array}{c}
\boldsymbol{\Phi}_{\boldsymbol{q}_{\mathrm{D}}^{\mathrm{T}}}^{\mathrm{T}} \\
\boldsymbol{\Phi}_{\boldsymbol{q}_{\mathrm{I}}}^{\mathrm{T}}
\end{array}\right]=0
\end{gathered}
$$

The direct calculation in Eq.(5) shows that $\boldsymbol{N}$ is the null space of $\boldsymbol{\Phi}_{q}$. So, pre-multiplication of Eq.(1) by $\boldsymbol{N}^{\mathrm{T}}$ can eliminate corresponding Lagrange multipliers $\lambda$ as[17]

$$
\boldsymbol{H}=\boldsymbol{N}^{\mathrm{T}} \boldsymbol{M} \ddot{\boldsymbol{q}}-\boldsymbol{N}^{\mathrm{T}} \boldsymbol{F}+\boldsymbol{N}^{\mathrm{T}} \boldsymbol{\Phi}_{\boldsymbol{q}}^{\mathrm{T}} \boldsymbol{\lambda}=\boldsymbol{N}^{\mathrm{T}} \boldsymbol{M} \ddot{\boldsymbol{q}}-\boldsymbol{N}^{\mathrm{T}} \boldsymbol{F}=0 \text { (6) }
$$

The null space of the constraint Jacobian is first premultiplied to the equations of motion to eliminate the Lagrange multipliers. However, the resulting differential equations are still functions of all coordinates, velocities, and accelerations. Therefore, the Eq.(6) are perturbed with respect to the variations of all coordinates, velocities, and accelerations, which are coupled by the constraints. Taking variation of Eq. (6) with $\boldsymbol{q}, \dot{\boldsymbol{q}}$ and $\ddot{\boldsymbol{q}}$ yields

$$
\delta \boldsymbol{H}=\boldsymbol{H}_{\boldsymbol{q}} \delta \boldsymbol{q}+\boldsymbol{H}_{\dot{q}} \delta \dot{\boldsymbol{q}}+\boldsymbol{H}_{\ddot{q}} \delta \ddot{\boldsymbol{q}}=\mathbf{0}
$$

Equation (7) can be rewritten in a matrix form as

$$
\left[\begin{array}{lll}
\boldsymbol{H}_{\boldsymbol{q}} & \boldsymbol{H}_{\dot{\boldsymbol{q}}} & \boldsymbol{H}_{\ddot{q}}
\end{array}\right]\left[\begin{array}{c}
\delta \boldsymbol{q} \\
\delta \dot{\boldsymbol{q}} \\
\delta \ddot{\boldsymbol{q}}
\end{array}\right]=0
$$

Since the coordinates, velocities, and accelerations are tightly coupled by the position, velocity, and acceleration level constraints, direct substitution of the relationships among these variables yields very complicated equations to be implemented. Variations of position, velocity and acceleration level constraints are

$$
\begin{gathered}
\boldsymbol{\Phi}_{q} \delta \boldsymbol{q}=0 \\
\dot{\boldsymbol{\Phi}}_{q} \delta \boldsymbol{q}+\boldsymbol{\Phi}_{q} \delta \dot{\boldsymbol{q}}=0 \\
\ddot{\boldsymbol{\Phi}}_{q} \delta \boldsymbol{q}+\dot{\boldsymbol{\Phi}}_{q} \delta \dot{\boldsymbol{q}}+\boldsymbol{\Phi}_{q} \delta \ddot{q}=0
\end{gathered}
$$

The perturbed constraint equations are then simultaneously solved for variations of all coordinates, velocities, and accelerations only in terms of the variations of the independent coordinates, velocities, and accelerations.

Appending the trivial identity relationships for the variations of independent coordinates, velocities and accelerations to Eq.(2) yields[17]

$$
\left[\begin{array}{ccc}
\boldsymbol{\Phi}_{q} & 0 & 0 \\
\dot{\boldsymbol{\Phi}}_{q} & \boldsymbol{\Phi}_{q} & 0 \\
\ddot{\boldsymbol{\Phi}}_{q} & \dot{\boldsymbol{\Phi}}_{q} & \boldsymbol{\Phi}_{q}
\end{array}\right]\left[\begin{array}{c}
\delta \boldsymbol{q} \\
\delta \dot{\boldsymbol{q}} \\
\delta \ddot{\boldsymbol{q}}
\end{array}\right]=\left[\begin{array}{ccc}
0 & 0 & 0 \\
0 & 0 & 0 \\
0 & 0 & 0 \\
& \boldsymbol{I} & \boldsymbol{I}
\end{array}\right]\left[\begin{array}{c}
\delta \boldsymbol{q}_{\mathrm{I}} \\
\delta \dot{\boldsymbol{q}}_{\mathrm{I}} \\
\delta \ddot{\boldsymbol{q}}_{\mathrm{I}}
\end{array}\right] \text { (10) }
$$


Equation (10) is solved for the $\delta \boldsymbol{q}, \delta \dot{\boldsymbol{q}}$ and $\delta \ddot{\boldsymbol{q}}$ substituted into the linearized equations of motion in Eq.(8) to yield the following linearized equations of motion only in terms of the variations of independent coordinates, velocities and accelerations:

$$
\begin{aligned}
{\left[\begin{array}{lll}
\boldsymbol{H}_{\boldsymbol{q}} & \boldsymbol{H}_{\dot{\boldsymbol{q}}} & \boldsymbol{H}_{\ddot{\boldsymbol{q}}}
\end{array}\right]\left[\begin{array}{lll}
\boldsymbol{\Phi}_{\boldsymbol{q}} & \\
\dot{\boldsymbol{\Phi}}_{\boldsymbol{q}} & \boldsymbol{\Phi}_{q} & \\
\ddot{\boldsymbol{\Phi}}_{\boldsymbol{q}} & \dot{\boldsymbol{\Phi}}_{\boldsymbol{q}} & \boldsymbol{\Phi}_{\boldsymbol{q}} \\
& \boldsymbol{I}
\end{array}\right]^{-1}\left[\begin{array}{ccc}
0 & 0 & 0 \\
0 & 0 & 0 \\
0 & 0 & 0 \\
& \boldsymbol{I}
\end{array}\right]\left[\begin{array}{c}
\delta \boldsymbol{q}_{\mathrm{I}} \\
\delta \dot{\boldsymbol{q}}_{\mathrm{I}} \\
\delta \ddot{\boldsymbol{q}}_{\mathrm{I}}
\end{array}\right]=0(11) } \\
\boldsymbol{M}^{*} \delta \ddot{\boldsymbol{q}}_{\mathrm{I}}+\boldsymbol{C}^{*} \delta \dot{\boldsymbol{q}}_{\mathrm{I}}+\boldsymbol{K}^{*} \delta \boldsymbol{q}_{\mathrm{I}}=0
\end{aligned}
$$

Direct comparison of Eq.(11) and Eq.(12) yields the $\boldsymbol{M}^{*} 、 \boldsymbol{C}^{*}$ and $\boldsymbol{K}^{*}$ matrices. Then calculate eigenvalues of the equivalent linear equations and nonlinear frequency with multibody dynamic contact, constraints and loads and so on.

The undamped equivalent linear equations can be written as

$$
\boldsymbol{M}^{*} \delta \ddot{\boldsymbol{q}}_{\mathrm{I}}+\boldsymbol{K}^{*} \delta \boldsymbol{q}_{\mathrm{I}}=0
$$

Then we get undamped eigenvalues equations as

$$
\boldsymbol{K}^{*} \boldsymbol{u}=\boldsymbol{\omega}^{2} \boldsymbol{M}^{*} \boldsymbol{u}
$$

$\mathrm{Eq}(12)$ and (14) is implemented simultaneously during the equations of motion for multibody systems Eq(1) is solved. As a result, the results of dynamics characteristics and nonlinear frequency of multibody contact dynamics systems can be achieved by dynamic simulation analysis.

\section{CONTACT NONLINEAR DYNAMICS SIMULATION OF NUMERICAL EXAMPLES}

Dynamics analysis and contact nonlinear frequencies of one ball or roller contact with two blocks are developed to demonstrate the validity of the proposed method. There are three kinds of nonlinear models discussed in the paper. First, one ball contact with two blocks model(called BCM), Second, one roller contact with two blocks model(called RCM), Third, two equivalent nonlinear springs connecting with two blocks model(called ESM).

\section{A. Contact dynamics models}

The BCM is showed in Fig1 and it is similar with part of a ball bearing. Considering dynamic contact between ball and triangle mesh elements of the surfaces, the contact dynamics model is established. The parameters is following as: two blocks mass $\mathrm{m}_{1}=\mathrm{m}_{3}=10 \mathrm{~kg}$, ball mass $\mathrm{m}_{2}=32.88 \mathrm{~kg}$, gravity acceleration $\mathrm{g}=9810 \mathrm{~mm} / \mathrm{s}^{2}$, two blocks height $\mathrm{h}_{1}=\mathrm{h}_{2}=100 \mathrm{~mm}$, ball radius $\mathrm{r}_{2}=100 \mathrm{~mm}$, block $\mathrm{m}_{3}$ velocity $\dot{y}_{3}=10 * \cos (168.7 \mathrm{t}) \mathrm{mm} / \mathrm{s}$, radial $\operatorname{load}(\mathrm{Y}) \mathrm{F}_{0}=-200 \mathrm{~N}$, Hertz contact stiffness $\mathrm{K}_{\mathrm{c} 21}=1 \mathrm{E} 6 \mathrm{~N} / \mathrm{mm}^{1.5}, \mathrm{~K}_{\mathrm{c} 23}=9.2 \mathrm{E} 5 \mathrm{~N} / \mathrm{mm}^{1.5}$, contact damper $\mathrm{C}_{\mathrm{c}}=1 \mathrm{~N} . \mathrm{s} / \mathrm{mm}$.

The friction, initial clearance are ignored and the block $\mathrm{m}_{1}$ is fixed, the block $\mathrm{m}_{3}$ has $\mathrm{Y}$ translational joint and velocity $\dot{y}_{3}$ along Y. Then, considering initial conditions, $\mathrm{Eq}(14)$ is applied to calculate the undamped frequencies. There are six freedoms of the BCM, five directions $(0 \mathrm{~Hz})$ free rigid vibration and one undamped frequency $(149.7 \mathrm{~Hz})$. It's the start point of nonlinear frequency and vibrating along Y direction shown in Fig3a.

In figure 2, the vibration displacements and normal contact force of the BCM are periodically variational except for the short start moment. In figure 3a, the contact nonlinear frequencies of the BCM and RCM are also periodically nonlinear and time-varying. Because the initial contact state of the BCM is same as the state of undamped system, the start value of nonlinear frequency of the BCM is the undamped frequency $(149.7 \mathrm{~Hz})$. The results of nonlinear frequencies at certain state can be validated using Gupta and other classical methods[4,6].

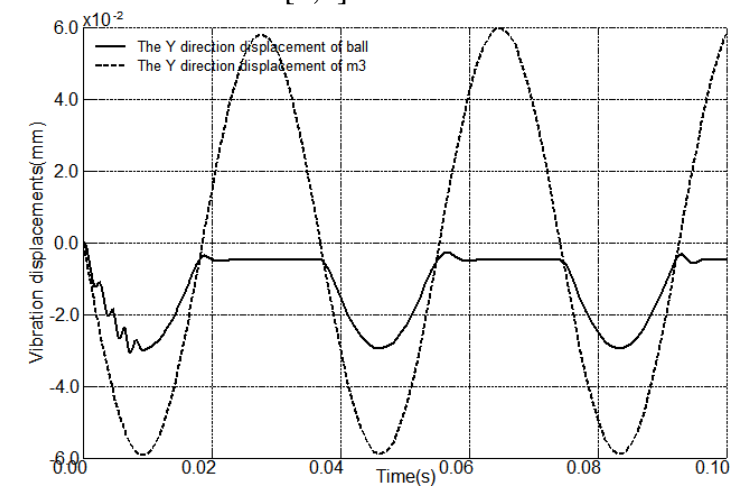

(a)The displacements of ball and block m3

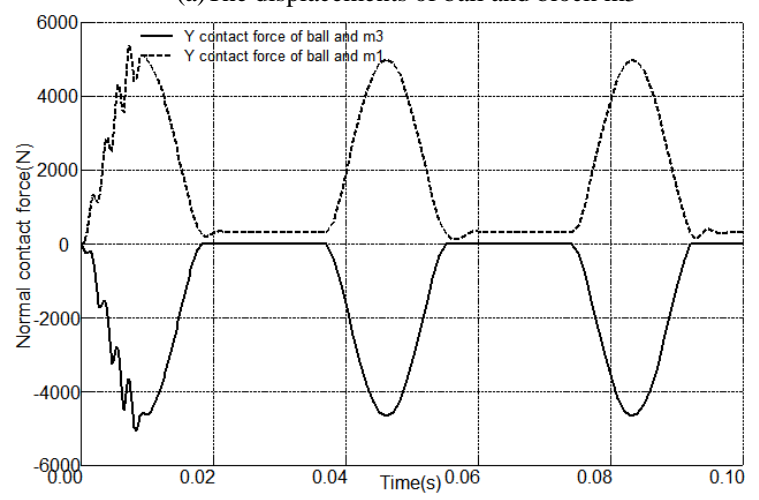

(b)The dynamic contact force

Fig2.The vibration displacements and normal contact force of one ball and two blocks model

Comparing analysis with figure 2 and 3a, under the conditions of $\dot{y}_{3}$ and $\mathrm{F}_{0}=-200 \mathrm{~N}$, constrainted block $\mathrm{m}_{3}$ is going to contact dynamically with ball. The dynamic contact forces of the BCM are increasing with the increment of contact elastic deformation in $\mathrm{Y}$ direction. The rule of nonlinear frequency is $1 / 6$ power function of contact force[6]. It indicates the enhanced stiffness of the BCM when the nonlinear frequency is increasing, vice versa. It's well known that the contact boundaries are unilateral constraints. When the ball contacts with block $\mathrm{m}_{1}$ only by gravity and departs from $\mathrm{m}_{3}$, the contact nonlinear 
frequency is a constant value shown in figure 3a. The dynamic forces are also keeping constant at this moment, shown in figure $2 b$.

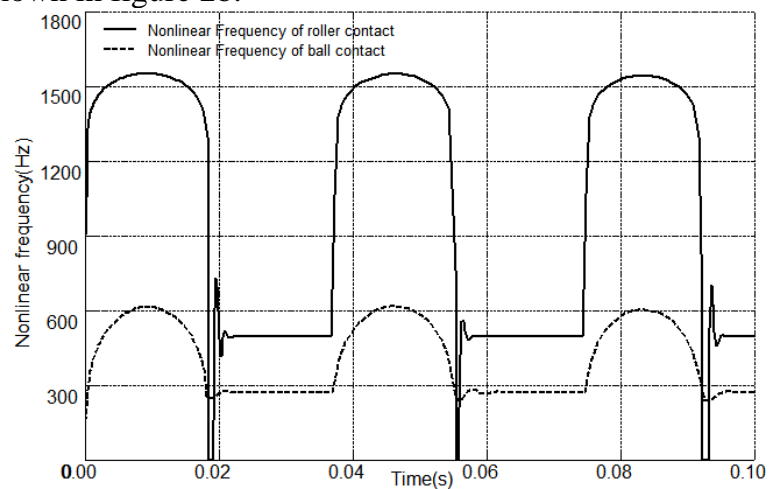

(a)nonlinear frequency

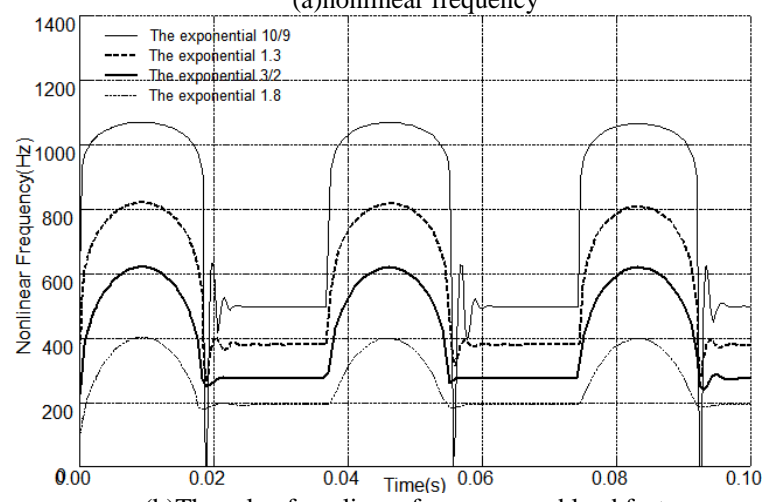

(b)The rule of nonlinear frequency and load factor Fig3.The contact nonlinear frequencies

In figure 3a, the nonlinear frequency of the RCM is higher, the bandwidth is wider and the peak is smoother than that of the BCM under the same conditions. When the roller departs from $\mathrm{m}_{3}$, it loses contact with block $\mathrm{m}_{1}$ instantaneously because of the vibration and contact-impact. The frequency of the RCM will be reduced to zero at this moment. In figure $3 \mathrm{~b}$, the exponential or load-deformation factor has an important effect on the nonlinear frequency of multibody systems. When the load-deformation factor is increasing, the nonlinear frequency is lower, the bandwidth is narrower and the peak is sharper of neighbor peaks and valley. As a result, the nonlinear characteristics of the frequency become stronger synchronously. It suggests that the stronger contact nonlinearity actually reduce the whole stiffness of multibody systems.

The calculated results show that the frequencies are constant values only when the contact states of multibody systems are invariable, otherwise, the frequencies are nonlinear, time-varying based on the boundaries and states of multibody systems. Therefore, the contact nonlinear frequency of the BCM and RCM presents a periodic bandwidth with double peak values based on the distance of neighbor peaks and valley.

\section{B. Equivalent spring-damper models}

The model(The ESM) shown in Fig1 is connected by two equivalent nonlinear spring-dampers between ball center and two blocks centers instead of dynamic contact and solved by the proposed method. In figure 4, the frequency is a constant value when the spring-dampers are linear, because the ESM becomes a linear multibody system. The frequencies are periodic, nonlinear, time-varying when the spring-dampers are nonlinear. The exponential 1.5 of the spring-dampers is simulated ball contact and exponential 1.1 simulated roller contact.

According to the results, the nonlinear frequencies of the ESM are only part of the frequencies of the BCM and RCM nearby the high peaks, especially the exponential 1.1 is simulated roller contact. The equivalent spring-dampers are always connected with two blocks, which are bilateral constraints. So, the lower valley of the nonlinear frequencies is especially different from the BCM and RCM. As a result, it's particularly cautious to use equivalent nonlinear springdampers instead of the dynamic contact to understand the system behavior of a contact nonlinear multibody system, especially for the strong contact nonlinearity like ball bearings and roller bearings.

In figure 5, the Poincare of the ESM is a close curve, it's quasi periodicity. The Poincaré of the BCM is chaotic phenomena.
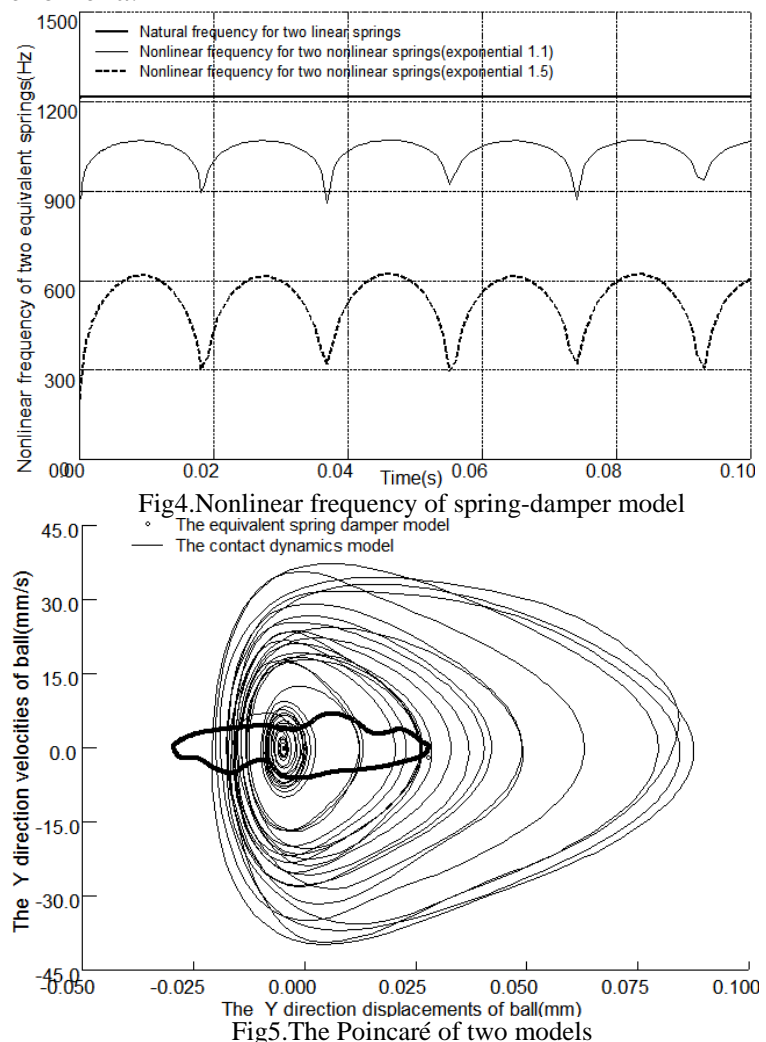


\section{CONCLUSIONS}

The models of contact nonlinear dynamics and equivalent spring-damper of a simple multibody system have been developed using dynamic simulation analysis. The contact frequencies calculated by the linearization method proposed in the paper are nonlinear, time-varying, a periodic bandwidth with high and low peaks, which is based on the boundaries and states of multibody systems. The nonlinear frequencies of the ESM are different from the BCM and RCM, especially the lower valley because the equivalent spring-dampers are always bilateral constraints.

The linearization method and the models in the paper are general and available to research the contact nonlinear frequencies of rolling bearings, as long as the corresponding contact dynamics models can be accurately constructed for rolling bearings. The consideration of clearance, complex loads and more motion DOFs in the simulation study makes necessary a more detailed analysis in future models and studies.

\section{ACKNOWLEDGMENT}

The authors would thank the funds provided by the Chinese Government through the Project NSFC-11002062 and the Yunnan Provice though the Project NSFC.

\section{REFERENCES}

[1] P.Eberhard, W.Schiehlen, "Computational Dynamics of Multibody Systems: History, Formalisms and Applications," Journal of Computational and Nonlinear Dynamics, 2006,1,pp. $1-12$.

[2] G.Javierde, B.Eduardo, "KINEMATIC AND DYNAMIC SIMULATION OF MULTIBODY SYSTEMS: The RealTime Challenge” Springer-Verlag,1993.

[3] N.Iakov, "Modeling and Simulation of Contacting Flexible Bodies in Multibody Systems,"[PHD Thesis], LINKÖPINGS UNIVERSITET, 2002.
[4] T Q.Yao. "Research on Multibody Contact Dynamics of Ball Bearings" [PHD Thesis]. Kunming University of Science and Technology .2009.

[5] Y X.Chen, "Research on Vibrations of Cyclindrical Roller Bearings Based on Contact Mechanics,"[PHD Thesis], Huazhong University of Science and Technology .2005.

[6] Harris, Tedric A. and Michael N. Kotzalas. Rolling Bearing Analysis, Fifth Edition-2 Volume Set. Taylor \& Francis Group, LLC.2007.

[7] H V.Liew, T C.Lim, "Analysis of time-varying rolling element bearing characteristics," Journal of Sound and Vibration, 2005.283,pp.1163-1179.

[8] S P. Harsha, K.Sandeep and R. Prakash, "Non-linear Dynamic Behaviors of Rolling Element Bearings due to Surface Waviness,” Journal of Sound and Vibration, 2004. 272,pp.557-580.

[9] L C.Zhao, J J.Ma, “elastic contact vibrations of ball bearings," Chinese Journal of Mechanical Engineering, 2003, 39(5),pp.60-64.

[10] V N. Sohoni, J. Whitesell, "Automatic linearization of constrained dynamical models," ASME, Journal of Mechanisms, Transmissions, and Automation in Design 1986.108,pp.300-304.

[11] C P.Neuman, J J.Murray, "Linearization and Sensitivity Functions of Dynamic Robot Models," IEEE Transactions on Systems, Man, and Cybernetics, 1984,6(14),pp.805-818.

[12] C A.Balafoutis, P.Misra, R V.Patel, "Recursive Evaluation of Linearized Dynamic Robot Models," IEEE Journal of Robotics and Automation, 1986,3(2),pp.146-155.

[13] C.Gontier, Y.Li, "Lagrangian Formulation and Linearization of Multibody System Equations,” Computers \& Structures, 1995,2(57),pp.317-331.

[14] B. Minaker and P. Frise, "Linearizing the Equations of Motion for Multibody Systems Using an Orthogonal Complement Method," Journal of Vibration and Control, 2005,121pp. 51-66.

[15] A G.Lynch, M J.Vanderploeg, "A symbolic formulation for linearization of multibody equation of motion," ASME, Journal of Mechanical Design,1995,117,pp.441-445.

[16] ANSYS User's Manual, ANSYS Inc, 2010.

[17] RecurDyn User’s Manual, FunctionBay Inc, 2010. 